\title{
INFORMATIZAÇÃO DA ATENÇÃO BÁSICA A SAÚDE: AVANÇOS E DESAFIOS
}

\author{
Ricardo Bezerra Cavalcante ${ }^{1}$, Daniela Dias Vasconcelos², Tarcísio Laerte Gontijo ${ }^{3}$, Eliete \\ Albano de Azevedo Guimarães ${ }^{4}$, Richardson Miranda Machado ${ }^{5}$, Valéria Conceição de Oliveira ${ }^{6}$
}

RESUMO: Objetivo: analisar a implantação da estratégia e-SUS Atenção Básica na Região Oeste de Minas Gerais, Brasil. Método: estudo de caso, descritivo, com dados de 62 responsáveis pela implantação em 54 municípios, de maio a junho de 2015, além de dois entrevistados e análise dos documentos: "Implantação do e-SUS AB na SRS Divinópolis" e "Municípios por Situação de Implantação do e-SUS AB na SRS Divinópolis". Os dados foram analisados porestatística descritiva e Análise de Conteúdo, com suporte do software Atlas TI, versão 7.0. Resultados: em 44 (81,5\%) dos municípios foi implantado apenas o cadastro de dados simplificado e $22(40,7 \%)$ dos municípios estão no cenário três de implantação, 34 (54,8\%) dos responsáveis pela implantaçãonão são servidores públicos efetivos. Conclusão: a implantação apresenta fragilidades relacionadas à infraestrutura, deficiências nas capacitações e resistência dos profissionais. É necessário rever o planejamento da implantação considerando sua complexidade.

DESCRITORES: Sistema de informação em saúde; Tecnologia da informação; Informática em saúde; Informática em enfermagem; Atenção primária à saúde.

\section{COMPUTERIZATION OF PRIMARY HEALTH CARE INFORMATION SYSTEMS: ADVANCES AND CHALLENGES}

ABSTRACT: Objective: to analyze the implantation of the e-SUS Primary Health Care strategy in the West Region of Minas Gerais, Brazil. Method: descriptive case study, with data from 62 people responsible for implementation in 54 municipalities, between May and June 2015, as well as two interviewees and analysis of the following documents: "Implantation of the e-SUS PHC in SRS Divinópolis" (Implantação do e-SUS AB na SRS Divinópolis) and "Municipalities, by Implantation of the e-SUS PHC in SRS Divinópolis" (Municípios por Situação de Implantação do e-SUS AB na SRS Divinópolis). The data were analyzed by descriptive statistics and Content Analysis, using the Atlas ti software, version 7.0. Results: in 44 (81.5\%) of the municipalities, only the simplified data register was implemented, and $22(40.7 \%)$ of the municipalities are in scenario three of implantation, $34(54.8 \%)$ of those responsible for implantation are not permanently contracted staff. Conclusion: The implantation has weaknesses related to infrastructure and shortcomings in the professionals' training and resistance. It is necessary to review the planning of the implantation, taking its complexity into account.

DESCRIPTORS: Health Information Systems; Information Technology; Health Informatics; Nursing Informatics; Primary Health Care.

\section{INFORMATIZACIÓN DE LA ATENCIÓN PRIMARIA DE SALUD: AVANCES Y DESAFÍOS}

RESUMEN: bjetivo: Analizar la implantación de la estrategia del e-SUS Atención Primaria en la Región Oeste de Minas Gerais, Brasil. Método: Estudio de caso, descriptivo, con datos de 62 responsables de la implantación en 54 municipios, entre mayo y junio de 2015, además de dos entrevistados y análisis de documentos: "Implantação do e-SUS AB na SRS Divinópolis" y "Municípios por Situação de Implantação do e-SUS AB na SRS Divinópolis". Datos analizados por estadística descriptiva y Análisis de Contenido, con ayuda del software Atlas TI, versión 7.0. Resultados: En 44 (81,5\%) de los municipios se implantó sólo el registro de datos simplificado, $22(40,7 \%)$ de los municipios están en la etapa 3 de implantación, $34(54,8 \%)$ de los responsables de la implantación son empleados públicos efectivos. Conclusión: La implantación muestra debilidades relacionadas con infraestructura, capacitaciones deficientes y resistencia de los profesionales. Resulta necesario rever la planificación de la implantación, considerándose su complejidad.

DESCRIPTORES: Sistemas de Información en Salud; Tecnología de la Información; Informática en Salud; Informática Aplicada a la Enfermería; Atención Primaria de Salud.

\footnotetext{
${ }^{1}$ Enfermeiro. Doutor em Ciência da Informação, Pós-Doutorado em Ciência da Informação. Professor Adjunto da Universidade Federal de São João Del Rei. Divinópolis, MG, Brasil.

${ }^{2}$ Enfermeira. Mestranda no Programa de Pós-graduação em Enfermagem da Universidade Federal de São João Del Rei, Divinópolis, MG, Brasil.

${ }^{3}$ Enfermeiro, Doutor em Ciências da Saúde, Pós-Doutorado em Saúde Pública. Professor Adjunto da Universidade Federal de São João Del Rei, Divinópolis, MG, Brasil.

${ }^{4}$ Enfermeira. Doutora em Ciências da Saúde, Pós-Doutorado em Saúde Publica, Professor Adjunto da Universidade Federal de São João Del Rei, Divinópolis, MG, Brasil.

${ }^{5}$ Enfermeiro, Doutor em Psiquiatria. Professor Adjunto da Universidade Federal de São João Del Rei, Divinópolis, MG, Brasil.

${ }^{6}$ Enfermeira. Doutora em Enfermagem. Professor Adjunto da Universidade Federal de São João Del Rei, Divinópolis, MG, Brasil.
} 


\section{INTRODUÇÃO}

A informatização da Atenção Básica a Saúde (ABS) tem se conformado em vários países como importante estratégia para automatização de processos e qualificação da gestão da informação.(1) Estudos internacionais demonstram que a implantação bem-sucedida de tecnologias da informação na ABS fortalece o processo de trabalho e as práticas de cuidado. Também, aperfeiçoa a dispensação de medicamentos, a continuidade dos cuidados, a busca de informações e contribui para a segurança do paciente. ${ }^{(1-3)}$ Além disso, a informatização está relacionada à maior eficiência na recuperação dos registros clínicos e ao armazenamento de informações sobre as famílias e comunidades..$^{(1-3)}$

Entretanto, a informatização da ABS também tem se conformado como um desafio, principalmente em países em desenvolvimento como o Brasil, tendo em vista as fragilidades relacionadas à infraestrutura tecnológica, ao financiamento, à qualificação profissional e à organização de processos no contexto do Sistema Único de Saúde (SUS). ${ }^{(4-5)}$

O Ministério da Saúde brasileiro (MS), reconhecendo a necessidade de informatização da ABS, instituiu em 2013 a estratégia e-SUS Atenção Básica (e-SUS AB) com a intenção de reestruturar todas as informações da Atenção Básica (AB). Essa estratégia visa um SUS eletrônico, tendo como premissa contribuir para a gestão da informação produzida no processo de trabalho das equipes de ABS. ${ }^{(6)}$ Buscase implantar dois sistemas de software: o Coleta de Dados Simplificada (CDS) e o Prontuário Eletrônico do Cidadão (PEC). Busca-se também a disponibilidade de acesso à internet de alta performance, computadores e impressoras em todos os postos de trabalho, interconectados com outros pontos da rede assistencial. ${ }^{(6)}$

Dessa forma, a estratégia e-SUS AB tem como possíveis contribuições: a individualização dos dados de usuários e das atividades profissionais; a integração entre os diversos sistemas de informação do SUS; a eliminação do retrabalho no registro e a automação de processos; a produção de informações qualificadas para apoiar a gestão e o cuidado. ${ }^{(6)}$

As Diretrizes Nacionais de Implantação da Estratégia e-SUS AB preconizam a identificação de profissionais habilitados para a coordenação da implantação e a realização de diagnóstico da capacidade dos recursos humanos e tecnológicos. ${ }^{(6)}$ Busca-se, ainda, a integração do planejamento local ao Plano de Ação Regional, a qualificação de profissionais para a informatização e a viabilização de equipes de suporte. ${ }^{(6)}$ Sabe-se que tal informatização é parte do processo de reestruturação dos Sistemas de Informação em Saúde (SIS) instituídos pela Portaria n. ${ }^{\circ}$ 2.073/2011 que aponta anecessidade de definir sistemas a serem implementados e padronizados no Brasil. ${ }^{(7)}$

Não há uma análise diagnóstica publicizada do processo de informatização da ABS e, principalmente, a identificação dos desafios que emergem neste contexto. Assim, justifica-se a relevância e originalidade deste estudo. Nesse sentido, tem-se a seguinte questão norteadora: "Como o processo de implantação da estratégia e-SUS AB tem se desenvolvido na Região de Saúde Oeste de Minas Gerais?" Este estudo teve como objetivo analisar a implantação da estratégia e-SUS AB na Região de Saúde Oeste de Minas Gerais, Brasil.

\section{- MÉTODO}

Estudo de caso, descritivo, que se utilizou da triangulação em duas etapas: pesquisa de campo e pesquisa documental.

O uso da triangulação permite que o pesquisador faça a combinação dos requisitos dos métodos qualitativo e quantitativo.(8) A abordagem qualitativa justificou-se pela necessidade de apreender as subjetividades dos participantes relacionadas ao objeto. Já a abordagem quantitativa foi utilizada pela necessidade de caracterização dos participantes e extração de dados na pesquisa documental. 
O cenário de pesquisa foi a Região de Oeste de Minas Gerais, localizada no sudeste do Brasil, a qual se constitui de 54 municípios, em seis regiões de saúde, com população aproximada de 1,2 milhões de habitantes.

Na primeira etapa, no período de maio a julho de 2015, coletaram-se dados, por meio de questionário on-line, de todos os 108 responsáveis pelo processo de implantação da estratégia e-SUS AB nos 54 municípios da referida região. O questionário foi dividido em duas partes: caracterização dos participantes (idade, sexo, formação, ocupação, capacitação, vínculo empregatício e motivo da escolha como responsável); questões abertas sobre as ações desenvolvidas para implantação, dificuldades e estratégias para solucioná-las.

Foram feitas até três tentativas de convite, direcionados ao e-mail pessoal dos 108 potenciais participantes da pesquisa. A partir do terceiro convite, caso não respondido, o participante era excluído da pesquisa. Assim, 62 (57,4\%) participantes responderam o formulário on-line. Um participante, apesar de acessar o formulário, não aceitou participar do estudo.

O segundo instrumento para a coleta dos dados foi um roteiro de entrevista semiestruturado direcionado aos dois profissionais lotados na Superintendência Regional de Saúde (SRS), responsáveis pela coordenação da implantação na região em estudo. Contemplaram-se questões relacionadas à coordenação da implantação.

Na segunda etapa do estudo foi realizada uma pesquisa documental, que se atrelou à necessidade de identificar o status de implantação na região pesquisada no período de julho a setembro de 2015. Os dois documentos que compuseram o corpus da pesquisa foram: "Implantação da estratégia e-SUS AB na Superintendência Regional de Saúde (SRS) Oeste" e "Municípios por Situação de Implantação da estratégia e-SUS AB na SRS Oeste". Foram extraídas as seguintes informações: Sistemas de softwares da estratégia e-SUS AB instalados e Cenários de implantação da estratégia e-SUS AB.

Os dados provenientes da caracterização dos participantes e da pesquisa documental foram analisados por meio de estatística descritiva. Já os dados qualitativos foram analisados por meio da Análise de Conteúdo modalidade Temático-Categorial. ${ }^{(9)}$ Com vistas a sistematizar a organização e análise dos dados utilizou-se o software Atlas Ti, versão 7.0.

O estudo foi aprovado pelo Comitê de Ética em Pesquisa da Universidade Federal de São João Del Rei, sob parecer de n. 1.037 .609 .

\section{RESUlTADOS}

Verificou-se na pesquisa documental predomínio do software e-SUS AB, um sistema com Coleta de Dados Simplificada (CDS), registrado como instalado em 44 (81,5\%) dos municípios e 186 (58,4\%) das UBS, enquanto o sistema Prontuário Eletrônico do Cidadão (PEC) apareceu registrado como instalado em apenas $3(5,6 \%)$ dos municípios e $44(14 \%)$ do total de UBS (Tabela 1). Alguns municípios, seis $(11,1 \%)$ apresentam SIS próprios (sistemas desenvolvidos pelo município ou adquiridos de empresas privadas) integrados ao e-SUS AB, modalidade CDS. 
Tabela 1 - Sistemas de softwares do e-SUS AB instalados nos municípios e em Unidades Básicas de Saúde (UBS). Região de Saúde Oeste, MG, Brasil, 2015

\begin{tabular}{lcccc}
\hline & N.o de municípios & $\mathbf{\%}$ & N.o de UBS* & \% \\
\hline e-SUS AB CDS instalado & 44 & 81,5 & 186 & 58,4 \\
e-SUS AB CDS por meio de Sistema próprio & 6 & 11,1 & 80 & 25,1 \\
e-SUS AB PEC & 3 & 5,6 & 44 & 14 \\
Nenhum dos sistemas de softwares do e-SUS AB & 1 & 1,8 & 8 & 2,5 \\
Total & 54 & 100 & 318 & 100 \\
\hline
\end{tabular}

Fonte: Documento "Implantação do e-SUS AB na SRS Oeste"

* Número de Unidades Básicas de Saúde de todos os municípios

No que diz respeito aos cenários (Tabela 2) de implantação, os documentos analisados destacam que a maioria dos municípios se encontrava no cenário 1 (UBS não informatizada - apenas fichas de cadastro) e 2 (UBS sem internet, mas com e-SUS AB CDS), enquanto apenas $3(5,6 \%)$ dos municípios estavam no cenário 6 (UBS com internet estável e com a instalação apenas do e-SUS AB PEC), considerado o cenário mais avançado de implantação. Em relação aos cenários de implantação em que se encontravam as UBS dos municípios, predominava em 147 (46,2\%) das UBS o cenário 3. Em contrapartida, apenas 38 $(11,9 \%)$ das UBS estavam no cenário 6.

Tabela 2 - Cenários de implantação do e-SUS AB nos municípios e em Unidades Básicas de Saúde (UBS). Região de Saúde Oeste, MG, Brasil, 2015

\begin{tabular}{lcccc}
\hline & $\begin{array}{c}\text { N.o de } \\
\text { municípios }\end{array}$ & $\begin{array}{c}\text { \% } \\
\text { U.. } \mathbf{~ d e} \\
\text { UBS }\end{array}$ & \% \\
\hline Cenário 1: UBS não informatizada - apenas fichas de cadastro & 11 & 20,4 & 29 & 9,1 \\
Cenário 2: UBS sem internet, mas com e-SUS AB CDS & 14 & 25,9 & 60 & 18,9 \\
Cenário 3: UBS com internet e com e-SUS AB CDS & 22 & 40,7 & 147 & 46,2 \\
Cenário 4: UBS com internet limitada, e-SUS AB CDS e PEC & 4 & 7,4 & 44 & 13,8 \\
Cenário 5: UBS com internet limitada apenas com e-SUS AB PEC & 0 & 0 & 0 & 0 \\
Cenário 6: UBS com internet estável apenas com e-SUS AB PEC & 3 & 5,6 & 38 & 11,9 \\
Total & 54 & 100 & 318 & 100 \\
\hline
\end{tabular}

Fonte: Documento "Implantação do e-SUS AB na SRS Oeste"

* Número de Unidades Básicas de Saúde de todos os municípios

No que se refere à caracterização dos responsáveis pelo processo de implantação nos municípios, apresentaram em média 34 anos de idade e 16 anos de estudo. Dentre os graduados, 36 (72\%) possuíam formação em enfermagem. Sobre os cursos de pós-graduação lato sensu, a maioria era especialista na área de Atenção Básica/Saúde da Família e Gestão em Saúde Pública, enquanto nenhum participante apresentou especialização na área de tecnologia da informação (Tabela 3).

Tabela 3 - Caracterização dos profissionais responsáveis pelo processo de implantação do e-SUS AB. Região de Saúde Oeste, MG, Brasil, 2015. (continua)

\begin{tabular}{ccc}
\hline Variável & Número de profissionais & Porcentagem (\%) \\
\hline Cargo ocupado na SMS & & \\
Coordenação da AB & 38 & 61 \\
Responsável pelo SIS do município & 16 & 25,8 \\
Digitador & 05 & 8 \\
\hline
\end{tabular}




\begin{tabular}{|c|c|c|}
\hline Secretário(a) municipal de saúde & 02 & 3,2 \\
\hline Responsável técnico por processamento de dados & 01 & 1,7 \\
\hline \multicolumn{3}{|l|}{ Sexo } \\
\hline Feminino & 49 & 79 \\
\hline Masculino & 13 & 21 \\
\hline \multicolumn{3}{|l|}{ Escolaridade } \\
\hline Possuem graduação & 50 & 80,6 \\
\hline Não possuem graduação & 12 & 19,4 \\
\hline \multicolumn{3}{|l|}{ Pós-graduação lato sensu } \\
\hline Possuem especialização & 26 & 41,9 \\
\hline Não possuem especialização & 36 & 58,1 \\
\hline \multicolumn{3}{|l|}{ Vínculo empregatício } \\
\hline Servidor público efetivo & 28 & 45,2 \\
\hline Cargo comissionado & 17 & 27,4 \\
\hline Contratado & 15 & 24,2 \\
\hline Celetista & 01 & 1,6 \\
\hline Terceirizado & 01 & 1,6 \\
\hline \multicolumn{3}{|l|}{ Capacitação ofertada pela SES/SRS } \\
\hline Profissionais capacitados & 55 & 88,7 \\
\hline Profissionais não capacitados & 07 & 11,3 \\
\hline
\end{tabular}

A partir da análise dos dados qualitativos identificaram-se as categorias e subcategorias a seguir.

\section{Categoria 1: Ações desenvolvidas para implantação da estratégia e-SUS AB}

Na subcategoria "Ações voltadas para o cadastramento da população e adaptações no cotidiano de trabalho, viabilizando a implantaçãoe-SUS AB", inicialmente, o cadastramento da população e a utilização das fichas para esta finalidade foram reconhecidas como ações para possibilitar a implantação da estratégia e-SUS AB:

Os profissionais vêm trabalhando nas fichas individuais, procedimentos, atividade coletiva e individual odontológica para irem se habituando [...]. (E08)

A população está sendo cadastrada com as fichas do e-SUS e todos os atendimentos já estão sendo feitos através destas fichas. (E28)

Estes trechos elucidam a utilização das fichas que alimentam o sistema CDS; porém, nenhum entrevistado destacou o registro das informações no PEC.

O processo de cadastramento da população por meio das fichas tem desencadeado alguns efeitos sobre o cotidiano de trabalho.

[...] foi elaborado uma escala para dividir entre a assistência e o preenchimento das fichas[...].(E15)

Realizamos mutirões com a equipe para agilizar o processo acima citado. (E53)

Além disso, foi destacado o envolvimento de todos no preenchimento das fichas, a coexistência do registro manual e eletrônico, bem como o retrabalho e monitoramento das digitações.

As fichas de atividade coletiva, procedimentos e atendimentos têm sido preenchidas manualmente por todos profissionais [...] é a recepcionista da UBS que digita no sistema. (E39)

Fazemos o monitoramento da digitação na Coordenação da Atenção Primária por meio de verificação de fichas digitadas. (E38) 
Eu faço o acompanhamento semanal e mensal das fichas, correção com os profissionais em caso de erros. (E42)

Foi reconhecida a contratação de profissionais como um custo adicional e gerado devido à necessidade de cadastramento da população.

Tivemos que contratar um profissional para estar digitando o cadastro, gerando mais custo no município. (E37)

Mesmo com a contratação, ainda está insuficiente frente à demanda. (E47)

Os responsáveis pela implantação desenvolveram ações de conscientização dos profissionais de saúde na tentativa de motivá-los à cooperação neste processo.

[...] dialogar com os profissionais da equipe, enfatizando os benefícios do e-SUS, substituindo o SIAB, um programa tão arcaico e sem recursos. (E40)

As "Capacitações nas esferas Estadual/Regional e Municipal visando à implantação do e-SUSAB" conformaram outra subcategoria. Neste sentido, as capacitações foram reconhecidas como ações para viabilizar a implantação da estratégia e-SUS AB. Alguns entrevistados fizeram avaliação positiva das capacitações ofertadas pela SES/SRS; porém, outros relataram certa insatisfação com essa ação.

A capacitação foi proveitosa, instruiu eficientemente no processo de utilização do sistema. (E05)

A capacitação foi muito tensa, pois quem estava fazendo a capacitação não sabia como o novo sistema funcionava de fato. (E39)

Passam muito por cima, sem muito conhecimento pelo assunto. Pois não trabalham na prática. (E49)

Os entrevistados também destacaram que estão ocorrendo capacitações para os profissionais das UBS nos municípios, bem como outras ações instrutivas buscando viabilizar a implantação da estratégia e-SUS AB.

Estamos oferecendo capacitações aos profissionais da ESF (Estratégia de Saúde da Família) quanto à utilização e o funcionamento do sistema, e também para o preenchimento das fichas que são utilizadas no CDS. (E23)

[...] orientação junto aos profissionais das unidades da importância do e-SUS e da qualidade dos dados que são informados. (E30)

\section{Categoria 2: Dificuldades para implantação da estratégia e-SUS AB nos municípios}

Na primeira subcategoria "Tecnologia da informação imatura e coexistência com papel" os participantes destacaram questões que impactam negativamente na implantação da estratégia e-SUS $\mathrm{AB}$, bem como na assistência à população.

A maior dificuldade é o grande número de fichas, papéis que precisamos preencher que demanda muito tempo nosso, não tendo muito tempo para a assistência à comunidade. (E15)

As constantes atualizações do sistema CDS, ainda imaturo, também inviabilizam o processo de implantação:

O programa no começo era muito vago e não tinha como realizar o backup e com isso perdemos muitas fichas que foram digitadas e com mudança de versão não foram recuperadas. (E22)

Outra dificuldade apresentada pelos profissionais para implantação é a inadequação do CDS para as demandas por informações específicas, o que não instrumentaliza a atuação dos profissionais.

O programa ainda está no começo e não existe relatório para analisar as informações consolidadas. Não está nos ajudando a analisar as informações para atuarmos. (E10) 
Pode-se verificar, ainda, a insatisfação dos entrevistados acerca do desenvolvimento do software feito sem a participação de seus usuários, além da limitação do software de não permitir atualizações nos dados já cadastrados.

Infelizmente quem pensa na criação dos programas, na maioria das vezes, está longe da realidade. A construção é "de cima para baixo" e deveria ser o contrário. (E10)

[...] o programa não permite alterar dados já incluídos, [...] o que já foi enviado para o DATASUS fica perdido. (E10)

Na segunda subcategoria "As pessoas como barreiras ao processo de implantação" os entrevistados destacaram inicialmente que os profissionais apresentam resistências às mudanças:

Durante as reuniões para informação do processo para a equipe, muitos profissionais se mostram bastantes resistentes com a mudança. (E40)

A dinâmica da população na comunidade também foi reconhecida como uma dificuldade.

[...] como acrescentar um membro da família, excluir o usuário que faleceu, acrescentar uma condição de saúde?(E10)

A população do município muda com frequência de endereço. (E09)

Dificuldade para efetuar o cadastro dos residentes nas microáreas[...].(E31)

A última subcategoria "Recursos físicos e financeiros como obstáculos à implantação" destaca ausência de infraestrutura adequada para a demanda informacional e insatisfação com a forma em que o processo de implantação do sistema vem ocorrendo.

[...] não temos infraestrutura suficiente para a implantação do projeto, mas para o Ministério isso não é visto como empecilho, mas creio eu que é um dos maiores. (E17)

Esse novo sistema foi passado para os municípios sendo que o governo não informatizou as unidades [...]. (E47)

\section{DISCUSSÃO}

Apesar do estabelecimento de diretrizes nacionais( ${ }^{(6)}$ para a implantação da estratégia e-SUS AB, na prática, na região pesquisada, não há processo sistematizado e tais diretrizes não são devidamente aplicadas. Verificamos, na tabulação dos dados do questionário on-line que os profissionais responsáveis pela implantação, em sua maioria, não possui formação ou especialização relacionada à área de tecnologias da informação, não são efetivos em seus cargos e acumulam a coordenação da atenção básica, juntamente com a responsabilidade pela implantação em seus municípios.

Sendo assim, não há critérios para a escolha do responsável pela implantação da estratégia e-SUS AB. Sabe-se que a indefinição de papéis e atribuições na liderança do processo de informatização em saúde geram efeitos negativos sobre a organização do processo de trabalho. ${ }^{(10)}$ Além disso, o acúmulo de funções de profissionais fragiliza a organização de informações dentro da estrutura organizacional e estimula comportamentos prejudiciais para a gestão da informação. ${ }^{(11)}$ Nesse sentido, há preocupação legítima com a formação de recursos humanos, responsáveis por tecnologias da informação no contexto da saúde pública, o que pode ser um fator importante na subutilização de tais tecnologias. ${ }^{(10)}$

Em relação ao status da implantação, predominaram a instalação do CDS, os cenários 2 e 3 para as UBS e alguns municípios não iniciaram o processo de implantação. Triangulando os dados coletados, constatamos que apesar da instalação do PEC ter sido verificada na pesquisa documental, nas entrevistas não houve qualquer menção dos respondentes sobre sua instalação nos municípios.

Tais achados corroboram o estudo realizado no estado do Mato Grosso, que em 2015nenhum de seus municípios havia implantado o PEC. ${ }^{(12)}$ Este cenário constatado é bem divergente do proposto pelo Ministério da Saúde, que seria o cenário 6 de implantação, em que o PEC estaria em utilização nas UBS com internet estável. Ressalta-se que a utilização do PEC nas UBS representa a possibilidade de 
qualificação do registro dos cuidados prestados à população, bem como a continuidade da assistência dos mesmos, contribuindo para a gestão do cuidado.

Estudos internacionais corroboram com esses achados ao assinalar que a implementação de um prontuário eletrônico otimiza a integração de informações, facilitam o acesso simplificado aos cuidados, favorecem o diagnóstico precoce de doenças e qualificam as práticas de cuidado. ${ }^{(13-15)}$

Confirmando a constataçãoda implantação predominante do sistema de software CDS, o preenchimento de seus instrumentos de coleta foi reconhecido pelos participantes como uma das principais ações do processo de implantação e com efeitos significativos sobre o trabalho das equipes, tais como a sobrecarga, a redução do tempo para o cuidado, as imposições e resistências. Tais efeitos são advindos do preenchimento das fichas e configuram a coexistência entre papel e informatização. A coexistência entre papel e tecnologia aponta para a imaturidade da própria tecnologia ${ }^{(16)}$ e tende a influenciar negativamente o processo de trabalho, tornando-o ineficiente, lento e menos produtivo ${ }^{(17)}$.

Além disso, a implantação da estratégia e-SUS AB não deve ter como enfoque apenas o preenchimento de fichas, como determinação burocrática, mas o desenvolvimento da compreensão e da análise do que se coleta e como se pode utilizar destas informações para gerir o coletivo. Reconhecemos que as necessidades informacionais são influenciadas pela necessidade de cumprir as diretrizes de um programa ou política e de produzir informações sobre a produtividade e alcance de metas atreladas a financiamentos. ${ }^{(11)}$ Porém, para potencializar a utilização de tecnologias da informação, é necessário que a informação seja discutida, debatida e consensuada, a fim de obter maior potencial de retenção e compreensão. ${ }^{(18)}$

Ressaltamos que o cadastramento da população é tarefa atrelada ao sistema de informação anterior, a fim de se obter dados consolidados por meio de relatórios, e isso já ocorrerá. ${ }^{(16)}$ Os profissionais, no que tange à estratégia e-SUS AB, estão efetuando o cadastramento novamente, quando há inexistência de tecnologias capazes de fazer a transposição dos dados do sistema anterior. Isto configura o retrabalho, repete-se o equívoco de implantar tecnologias que não se adequam ao processo de trabalho; pelo contrário, espera-se que os profissionais e os processos sejam alterados/adaptados para que a tecnologia seja implantada. ${ }^{(10-11,16-17)}$ Isso revela um aspecto que necessita de atenção: o sistema deve atender as necessidades reais dos profissionais para não dificultar o processo de trabalho. ${ }^{(19)}$

A triangulação dos dados relacionados às capacitações dos responsáveis pela implantação da estratégia e-SUS AB revelou contradições. Os dados quantitativos demonstraram, de forma predominante, a realização de capacitações oferecidas pela SRS e SES. Porém, na percepção dos participantes, as capacitações da forma como aconteceram não são efetivas. Tais situações demonstram a necessidade de aperfeiçoar o processo de qualificação dos profissionais com vistas ao sucesso da implantação. Estudos nacionais e internacionais demonstram que a ausência de qualificação relacionada à tecnologia pode implicar em sua subutilização, baixa qualidade das informações coletadas, bem como análises dos dados insuficientes. ${ }^{(10-11,16-17)}$

É urgente a necessidade de um processo de capacitação sistemático e contínuo nas esferas de gestão local, municipal e regional/estadual. Na sua ausência, corre-se o risco de inviabilizar o processo de implantação, além de manter o status quo hegemônico no que tange à subutilização de sistemas de informação voltados para a gestão local..$^{(4,11,16)}$

Algumas dificuldades foram percebidas no processo de implantação: software inadequado às demandas informacionais; resistências dos profissionais; falta de infraestrutura das UBS; entre outras. Neste contexto, 'tecnologia, pessoas e infraestrutura' emergiram como dimensões que precisam ser valorizadas e repensadas no sentido de viabilizar o processo de implantação. É preciso desenvolver e implantar tecnologias da informação considerando as várias dimensões que as envolvem. ${ }^{(19)}$

Também, é preciso valorizar a infraestrutura da computação em hardware e software; conteúdo clínico; interface homem-computador; pessoas; fluxo de trabalho e comunicação; políticas internas governamentais, procedimentos e cultura; regras externas, regulamentos e pressões; mensuração e monitoramento constante. ${ }^{(19)}$ Não se devem empreender processos de implantação de tecnologias no contexto do SUS sem considerar estas dimensões, pois se corre o risco de desenvolvê-las com grande chance de fracasso. 
Em relação às limitações deste estudo, considera-se, inicialmente, a possibilidade de aplicabilidade de seus resultados apenas ao cenário analisado, não permitindo generalizações. Entretanto, ressaltamos que o processo de implantação da estratégia e-SUS AB vem ocorrendo em todo o país e pode apresentar situações semelhantes às verificadas nesta pesquisa. Assim, a originalidade e o ineditismo deste estudo podem contribuir para o dimensionamento de futuras avaliações da implantação em outras regiões brasileiras.

\section{CONCLUSÃO}

O processo de implantação da estratégia e-SUS AB, apesar de sua evolução, apresenta fragilidades relacionadas aos responsáveis por acompanhar a implantação, por ter infraestrutura insuficiente nas UBS, deficiências nas capacitações, resistência dos profissionais e efeitos negativos sobre o trabalho. Esses achados apontam para a necessidade de rever o planejamento do processo de informatização, com vistas a considerar as várias dimensões que a envolve. É preciso reconhecer politicamente a importância da informatização como suporte na organização do trabalho na atenção básica e a gestão do cuidado.

O uso da triangulação contribuiu para o fortalecimento das constatações, representadas por alinhamentos e contradições, possibilitando sucessivas aproximações à compreensão do objeto. Entretanto, reconhecemos a necessidade da continuidade de estudos relacionados à estratégia e-SUS $\mathrm{AB}$ e seus sistemas de software. É preciso avaliar os efeitos dos sistemas de softwaresobre a organização do trabalho e a gestão do cuidado. Além disso, a análise da utilização e aceitação destas tecnologias, sob a ótica dos profissionais, também se fazem necessárias.

\section{AGRADECIMENTO}

Esta pesquisa recebeu apoio financeiro da Fundação de Amparo à Pesquisa do Estado de Minas Gerais (FAPEMIG) e do Conselho Nacional de Desenvolvimento Científico e Tecnológico (CNPQ).

\section{- REFERÊNCIAS}

1. Montague $\mathrm{E}$. The promises and challenges of health information technology in primary health care. Primary Health Care Research Development. [Internet] 2014;15(3):227-30 [acesso em 21 fev 2018]. Disponível: https://doi.org/10.1017/ S1463423614000231.

2. Jeffries M, Phipps D, Howard RL, Avery A, Rodgers S, Ashcroft D. Understanding the implementation and adoption of an information technology intervention to support medicine optimisation in primary care: qualitative study using strong structuration theory. BMJ Open. [Internet] 2017;7:e014810 [acesso em 21 fev 2018]. Disponível: http://dx.doi. org/10.1136/bmjopen-2016-014810.

3. Rittenhouse DR, Ramsay PP, Casalino LP, McClellan Sean, Kandel ZK, Shortell MS. Increased Health Information Technology Adoption and Use Among Small Primary Care Physician Practices Over Time: A National Cohort Study. Annals of Family Medicine. [Internet] 2017;15(1):56-62. [acesso em 21 fev 2018]. Disponível: https://doi.org/10.1370/ afm.1992.

4. dos Santos AF, Fonseca SD, Araújo LL, Procópio CSD, Lopes EAS, Lima AMLD, Reis CMR, Abreu DMX, Jorge AO, Matta-Machado AT. Incorporação de Tecnologias de Informação e Comunicação e qualidade na atenção básica em saúde no Brasil. Cad. Saúde Pública. [Internet]. 2017;33(5):e00172815 [acesso em 20 fev 2018]. Disponível: http:// dx.doi.org/10.1590/0102-311x00172815.

5. Matta-Machado ATG, de Lima AMLD, de Abreu DMX, Araújo LL, Sobrinho DF, Lopes EAS, Teixeira GHS, dos Santos AF. Is the Use of Information and Communication Technology Associated With Aspects of Women's Primary Health Care in Brazil? J. Ambul. Care Manage. [Internet] 2017;40(2):49-59 [acesso em 20 fev 2018]. Disponível: http://dx.doi. org/10.1097/JAC.0000000000000187. 
6. Ministério da Saúde (BR). Secretaria de Atenção à Saúde. Departamento de Atenção Básica. Diretrizes Nacionais de Implantação da Estratégia e-SUS Atenção básica. [Internet] Brasília: Ministério da Saúde; 2014 [acesso em 10 dez 2014]. Disponível: http://189.28.128.100/dab/docs/portaldab/publicacoes/diretrizes_nacionais_esus.pdf.

7. Ministério da Saúde (BR). Portaria n.o 2.073, de 31 de agosto de 2011. Regulamenta o uso de padrões de interoperabilidade e informação em saúde para sistemas de informação em saúde no âmbito do Sistema Único de Saúde, nos níveis Municipal, Distrital, Estadual e Federal, e para os sistemas privados e do setor de saúde suplementar. Diário Oficial da União. [Internet] 31 ago 2011 [acesso em 10 dez 2014]. Disponível: http://bvsms.saude.gov.br/bvs/ saudelegis/gm/2011/prt2073_31_08_2011.html.

8. Marcondes NAV, Brisola EMA. Análise por Triangulação de Métodos: um referencial para pesquisas qualitativas. Rev. Univap. [Internet] 2014;20(35):201-08 [acesso em 13 dez 2014]. Disponível: http://dx.doi.org/10.18066/revunivap. v20i35.228.

9. Bardin, L. Análise de Conteúdo. Lisboa: Edições 70; 2013.

10 .Seitio-Kgokgwe O, Gauld RDC, Hill PC, Barnett P. Development of the National Health Information Systems in Botswana: Pitfalls, prospects and lessons. Online J. Public. Health Informatics. [Internet]. 2015;7(2) [acesso em 17 dez 2014]. Disponível: http://dx.doi.org/10.5210/ojphi.v7i2.5630.

11. Cavalcante RB, Watanabe YJA, Guimarães EAA, Gontijo TL, de Oliveira VC, Vasconcelos DD. Comportamento informacional de gestores da rede Hiperdia Minas.Perspectivas em Ciência da Informação. [Internet] 2017;22(3):33-55. [acesso em 19 fev 2018]. Disponível: http://dx.doi.org/10.1590/1981-5344/2734.

12. Astolfo S, Kehrig RT. O processo de implantação de uma estratégia integrada de SIS na APS no Mato Grosso, Brasil. Rev. Saúde Col. UEFS. [Internet] 2017;7(1):8-15 [acesso em 19 fev 2018]. Disponível: http://dx.doi.org/10.13102/ rscdauefs.v7i1.1169.

13. Huang MZ, Gibson CJ, Terry AL. Measuring Electronic Health Record Use in Primary Care: A Scoping Review. Appl. Clin. Inform. [Internet] 2018;9(1):15-33. [acesso em 20 fev 2018]. Disponível: http://dx.doi.org/10.1055/s-0037-1615807.

14. Gregory ME, Russo E, Singh H. Electronic Health Record Alert-Related Workload as a Predictor of Burnout in Primary Care Providers. Applied. Clin. Inform. [Internet] 2017;8 (3):686-97. [acesso em 21 fev 2018]. Disponível: https://doi. org/10.4338/ACl-2017-01-RA-0003.

15. Konerman MA, Thomson M, Gray K, Moore M, Choxi H, Seif E, Lok ASF. Impact of an electronic health record alert in primary care on increasing hepatitis c screening and curative treatment for baby boomers. Hepatology. [Internet] 2017;66 (6):1805-13. [acesso em 21 fev 2018]. Disponível: https://doi.org/10.1002/help.29362.

16. Carreno I, Moreschi C, Marina B, Hendges DJB, Rempel C, de Oliveira MMC. Análise da utilização das informações do Sistema de Informação de Atenção Básica (SIAB): uma revisão integrativa.Ciênc. saúde coletiva. [Internet] 2015;20(3):947-56. [acesso em 03 jan 2015]. Disponível: http://dx.doi.org/10.1590/1413-81232015203.17002013.

17. Vest J, Issel LM, Lee S. Experience of Using Information Systems in Public Health Practice: Findings from a Qualitative Study. Online J. Public Health Informatics. [Internet] 2014;5(3) [acesso em 03 jan 2015]. Disponível: http://dx.doi. org/10.5210/ojphi.v5i3.4847.

18. Long AC, Curtis JR. Enhancing informed decision making: is more information always better? Critical Care Medicine. [Internet]2015;43(3):713-14. [acesso em 19 fev 2018]. Disponível: http://dx.doi.org/10.1097/CCM.0000000000000797.

19. Sittig DF, Hardeep S. A New Socio-technical Model for Studying Health Information Technology in Complex Adaptive Healthcare Systems. Qual. Saf. Health Care. [Internet] 2010;19 (Suppl 3):68-74. [acesso 12 abr 2015]. Disponível: http://dx.doi.org/10.1136/qshc.2010.042085. 